$\left|\frac{124}{1 / 17 T^{8}}\right|_{\text {LA-7070-MS }}$

Informal Report
16.175

UC-34c

lssided: December 1977

\title{
Retrieval of the Energy Spectrum in Neutron TOF Measurements
}

Huan Lee

J. J. Walker

$+$

Ios Nalamos

solontific laboratory

of the University of California

LOS ALAMOS, NEW MEXICO O75AS

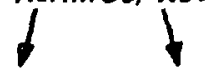

An Allirmative Action/Equal Opportunity Employer 


\title{
RETRIEVAL OF THE ENERGY SPECTRUM IN NEUTRON TOF MEASUREMENTS
}

by

Huan Lee and J. J. Walker

\begin{abstract}
In this report we investigate the problem of retrieving the time dependence of the neutron energy spectrum from the detector current data in the Faraday-cup experiments. The time-dependent energy spectrum can be retrieved only when the neutron source in question meets a certain criterion. We present a decailed analytic formulation for the folding process. We also spe1! out in detail the method that can be used to unfold the data in the retrievable case.
\end{abstract}

\begin{tabular}{|c|}
\hline 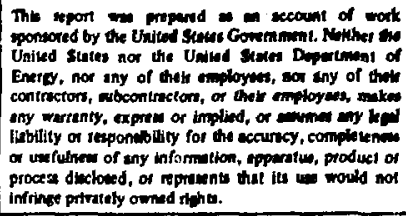 \\
\hline
\end{tabular}

\section{INTRODUCTION}

The measurement of the energy spectrum and the time history of a device's output neutrons provide information of considerable importance to the design community. The usual experimental setup for neutron measurements involves timeof-flight (TOF) techniques and the conversion of the neutrons to charged particles and the subsequent measuring of either the charge or the energy deposition of the latter. In Faraday-cup experiments, neutrons are converted into protons by passing through a suitable converter foil (CF). Since both the TOF effect and the energy loss of the prosons in passing through the CF inevitably modify the energy and time dependence of the particle beam, the primary task is how to retrieve information from the data in the form of detector current. In this report we discuss the effects due to TOF and energy loss of the protons in detail and present a general mathematical framework to fold the source neutron flux into detector current. We also examine the conditions under which the data can be unfolded back to the energy spectrum of the source neutrons.

The geometry of the experimental apparatus relevant to our problem is discussed in Sec. II. The effect of TOF to the particle beam is given in Sec. III. 
Section IV is devoted to the n-p elastic scattering, and Sec. $V$ deals with the problem of energy loss of the protons inside the CF. In Sec. VI we discuss various approximations and derive the formula for computing the energy spectrum of the neutron source with a $\delta$-function time dependence. Finally, in Sec. VII we illustrate how to unfold the data if the neutron source is described by a function separable in its time- and energy dependence.

\section{GEOMETRY}

We choose oúr coordinates so that the incoming neutron beam is para1le1 to the z-axis, and the converter foil lies in the $x-y$ plane as shown in Fig. 1. The vector $\vec{D}$, which connects the centers of the CF and the threshold foil (TF), is in the $x-z$ axis plane at an angle $\alpha$ relative to the $z$-axis. The TF is oriented to be perpendicular to $\vec{D}$.

Let $\vec{R}_{1}\left(\vec{R}_{2}\right)$ be the vector that specifies the location of an area element $d A_{1}\left(d_{2}\right)$ in the converter (threshold) foil. It is convenient to introduce polar coordinates for $\vec{R}_{1}$ and $\vec{R}_{2}$ in their respective foil planes. The polar angle $\theta_{1}$ of $\vec{k}_{1}$ is defined with respect to the $x$-axis, while the polar angle $\theta_{2}$ of $\vec{k}_{2}$ is defined with respect to $x^{\prime}$-axis, which is obtained by rotating the $x$-axis around the $y$-axis by an angle $\alpha$. From Fig. 2 it is easily seen that the vector $\vec{R}_{2}$ can be obtained by rotating the vector $\vec{R}_{0}$ around the $y$-axis by an angle $\alpha$. The Cartesian components of $\vec{R}_{1}$ and $\vec{R}_{2}$ are

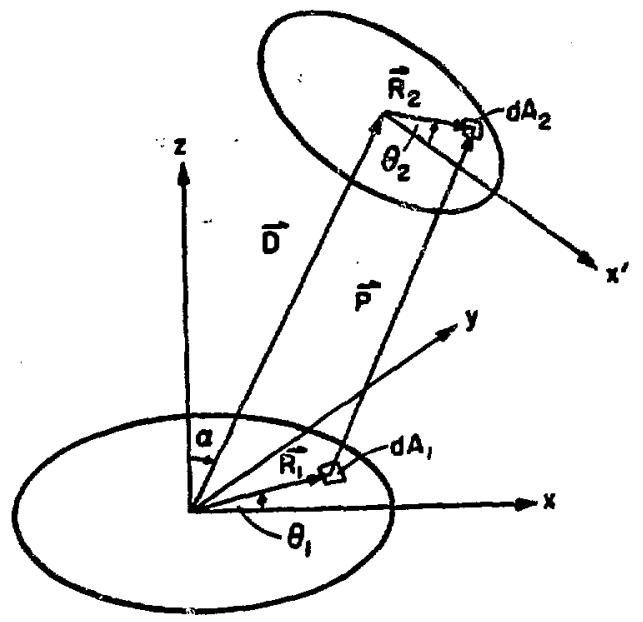

Fig. 1 .

Geometry describing the relative orientation between the converter foll and the threshold foil (or detector).

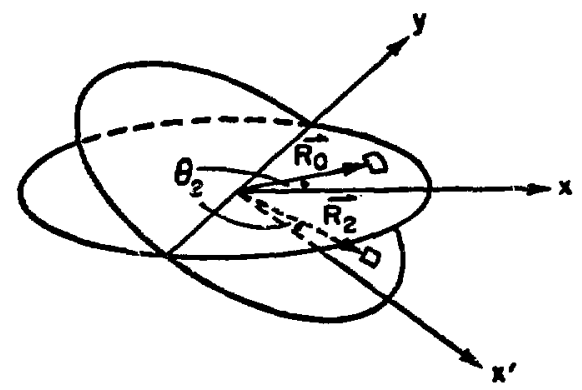

Fig. 2 .

Rotation that brings $\vec{R}_{0}$ into $\vec{R}_{2}$. 


$$
\vec{R}_{1}=\left(R_{1} \cos \theta_{1}, R_{1} \sin \theta_{1}, 0\right),
$$

and

$$
\begin{aligned}
\vec{R}_{2} & =R_{y}(\alpha) \vec{R}_{0} \\
& =\left(R_{2} \cos \theta_{2} \cos \alpha, R_{2} \sin \theta_{2},-R_{2} \cos \theta_{2} \sin \alpha\right),
\end{aligned}
$$

where $\vec{S}_{0}=\left(R_{2} \cos \theta_{2}, R_{2} \sin \theta_{2}, 0\right)$ and

$$
R_{y}(\alpha)=\left(\begin{array}{ccl}
\cos \alpha & 0 & \sin \alpha \\
0 & 1 & 0 \\
-\sin \alpha & 0 & \cos \alpha
\end{array}\right) \text {. }
$$

From

$$
\vec{D}=(D \sin \alpha, 0, D \cos \alpha)
$$

we can express $\vec{p}$ explicitly as

$$
\begin{aligned}
\overrightarrow{\mathbf{P}} & =\overrightarrow{\mathrm{D}}+\vec{R}_{2}-\overrightarrow{\mathrm{R}}_{1} \\
& =\left\{\begin{array}{l}
D \sin \alpha+\mathrm{R}_{2} \cos \theta_{2} \cos \alpha-\mathrm{R}_{1} \cos \theta_{1} \\
\mathrm{R}_{2} \sin \theta_{2}-\mathrm{R}_{1} \sin \theta_{1} \\
D \cos \alpha-\mathrm{R}_{2} \cos \theta_{2} \sin \alpha
\end{array}\right\}
\end{aligned}
$$

For fixed values of $D$ and $\alpha, \vec{p}$ is specified by the parameters $\left(R_{1}, \theta_{1}\right)$ and $\left(R_{2}\right.$, $\theta_{2}$ ). Using the symmetry of the setup, the range of parameters we have to consider are:

and

$$
\begin{aligned}
& 0 \leq R_{1} \leq r_{1}, \\
& 0 \leq \theta_{1} \leq \pi, \\
& 0 \leq R_{2} \leq r_{2}, \\
& 0 \leq \theta_{2} \leq 2 \pi,
\end{aligned}
$$

where $r_{1}\left(r_{2}\right)$ is the radius of the CF (TF). 
The area elementa $\mathrm{dA}_{1}$ and $\mathrm{dA}_{2}$ can be written as

$$
d A_{1}=R_{1} d R_{1} d \theta_{1} ; \quad 1=1,2
$$

Since the CF is very thin, the thickness dependence in the geometrical consideration discussed above can be ignored. However, the energy loss of the protons emitted inside and traveling through the CF depends on the pathlength. The pathlength of a proton emitted in a layer at a depth $\ell$ below the top surface of the foil and along the direction $\vec{p}$ is given by

$$
s=\frac{\ell p}{\mathbf{p}_{\mathbf{z}}}=\frac{\ell}{\cos \theta}
$$

where $\theta$ is the scattering angle of the proton with respect to the incoming neutron direction.

\section{TIME-OF-FLIGHT EFFECT}

Since the time-of-flight depends on the particle velocity, the time-dependent energy distribution (TED) of the neutron $f l u x$, denoted by $S_{0}(E, t)$, will be different from the TED of the neutron flux arriving at the foil, which we denote by $S(E, t)$. We use $E$ to stand for kinetic energy. Let $d$ be the distance between the source and the CF. A neutron of energy $E$ and velocity $v(E)$ leaving the source at $t=t^{\prime}$ will arrive at the foil $d / v$ later in time. This implies that for a fixed value $E, S(E, t)$ is simply the same as $s_{0}\left(E, t^{\prime}\right)$ evaluated at an earlier time

$$
t^{\prime}=t-\frac{d}{v(E)}
$$

Hence, the general relationship between $S$ and $S_{0}$ is

$$
S(E, t)=s_{0}\left(E, t \cdot \frac{d}{v(E)}\right)
$$

where $v$ is related to $E$ by 


$$
v(E)=\frac{\sqrt{E\left(E+2 m c^{2}\right)}}{E+m c^{2}} c \text {, }
$$

with $m$ being the neutron rest mass and $c$, the velocity of light.

We shall in the next section consider the TED of the protons produced inside the CF by n-p scattering.

\section{NEUTRON-PROTON SCATTERING}

Since the CF is very thin, the attenuation of the neutron flux passing through it can be neglected. Hence, we can consider the protons to be produced uniformly throughout the foil. We now make the following definitions:

$\mathrm{N}=$ hydrogen number density in the $\mathrm{CF}$,

$\frac{d \sigma}{d \Omega}\left(E_{0}, \theta\right)=$ differential cross section for $n-p$ elastic scattering with incoming neutron energy $\mathrm{E}_{0}$ and outgojing proton sccttered at an angle $\theta$, $F(E, t, \theta)=$ number of protons produced of energy $E /$ unit volume/solid angle/ unit time.

In $n-p$ elastic scattering, the energy of the outgoing proton $E$ is completely determined by $E_{0}$ and $\theta$. Neglecting the small mass difference between the proton and the neutron, we can show that

$$
E=\frac{2 m c^{2} E_{0} \cos ^{2} \theta}{2 m c^{2}+E_{0} \sin ^{2} \theta}
$$

In the rarge of $E_{0}$ of our practicei interest, $\frac{E_{0}}{2 m c^{2}} \leq 10^{-2}$; using the nonrelativistic limit of $\mathrm{Eg}$. (10),

$$
E=E_{0} \cos ^{2} \theta
$$

will only introduce an exror less than one percent. Following from their definition, we can write 


$$
F(E, t, \theta) d E=N \frac{d \sigma}{d \Omega}\left(E_{0}, \theta\right) S\left(E_{0}, t\right) d E_{0}
$$

Substituting Eq. (11) Into Eq. (12) we get

$$
F(E, t, \theta)=N \frac{d \sigma}{d \Omega}\left(\frac{E}{\cos ^{2} \theta}, \theta\right) s\left(\frac{E}{\cos ^{2} \theta}, t\right) \frac{1}{\cos ^{2} \theta} .
$$

If the use of Eq. (10) is desirable, $\mathrm{dE}_{0}$ in Eq. (12) can be replaced by

$$
\mathrm{dE}_{0}=\frac{4 \mathrm{~m}^{2} c^{4} \cos ^{2} \theta}{\left(2 m c^{2} \cos ^{2} \theta-E \sin ^{2} \theta\right)^{2}} \mathrm{dE} .
$$

\section{ENERGY LOSS OF PROTONS}

The protons, in passing through the $\mathrm{CF}$, will experience multiple Coulomb scattering with atomic electrons and thus lose energy. As the protons are deflected at each collision, the energy loss, as well as the angle of emergence, depends on the actual pathlength traveled. A Monte Carlo method is the best way to keep track of each individual pathlength and also to calculate the proton energy 10ss. In the following discussion we assume the $C F$ is thin enough and the protons are energetic enough so that each pathlength is essentially a straight line along the initial direction of motion of the proton. This assumption is quite reasonable since small angle scattering would be predominant. Let $L(E)$ be the energy loss function per unit length, i:e.,

$$
\frac{d E}{d s}=-L(E)
$$

Defining a function $h(E)$ so that

$$
\frac{d h}{d E}=1 / L(E),
$$

we Integrate Eq. (15) from $E=E_{1}$ to $E=E_{f}$ as 


$$
h\left(E_{i}\right)-h\left(E_{f}\right)=s
$$

The energy loss $\Delta \equiv E_{i}-E_{f}$ is clearly a function of pathlength $s$ and either initial energy $E_{i}$ or final energy $E_{f}$. It is important to notice that even at a fixed incoming neutron energy and scattering angle $\theta$, the protons emerging from the foil will have a spread in energy, buause they travel through different material thicknesses. We now proceed to examine how the energy spectrum of the protons created uniformly inside the foil is changed after suffering energy losses in passing through the foil.

Consider a proton of energy $E_{i}$ created at a depth $\ell$ beneath the foil top and traveling along $\theta$. Its pathlength through the foil is $s=\ell / \cos \theta$. In particular, the protons in the energy band $\mathrm{dE}_{i}$ will emerge from the foil in an energy band $\mathrm{dE}_{\mathrm{f}}$. Their relationship can be obtained by differentiating $\mathrm{Eq.} \mathrm{(17)} \mathrm{with} \mathrm{s}$ being held fixed, thus

$$
d E_{i} / L\left(E_{i}\right)=d E_{i} / L\left(E_{F}\right)
$$

Denoting the TED of the proton flux per unit solid angle leaving the $C F$ originating from a depth between $\ell$ and $\ell+d \ell$ as $G_{\ell}\left(E_{f}, t, \theta\right)$, we obtain

$$
G_{\ell}\left(E_{f}, t, \theta\right) d E_{f}=F\left(E_{i}, t, \theta\right) d E_{i} .
$$

Hence,

$$
G_{\ell}\left(E_{f}, t, \theta\right)=F\left(E_{i}, t, \theta\right) L\left(E_{i}\right) / L\left(E_{f}\right)
$$

The proton flux at a fixed $E_{f}$ emerging from the foil from all depths is given by

$$
\begin{aligned}
G\left(E_{f}, t, \theta\right) & =\int_{0}^{\delta} G_{\ell}\left(E_{f}, t, \theta\right) d \ell \\
& =\int_{E_{f}}^{E_{f}+\Delta\left(E_{f}, \delta, \theta\right)} G_{\ell}\left(E_{f}, t, \theta\right)\left(\frac{\partial \ell}{\partial E_{i}}\right)_{E_{f}} d E_{i},
\end{aligned}
$$


where $\delta$ is the thickness of the $C F$ and

$$
\Delta\left(E_{f}, \delta, \theta\right)=h^{-1}\left(h\left(E_{f}\right)+\delta / \cos \theta\right)-E_{f}
$$

From Eq. (17) and recalling $s=\ell / \cos \theta$, we find

$$
\left(\frac{\partial \ell}{\partial E_{i}}\right)_{E_{f}}=\cos \theta / L\left(E_{i}\right)
$$

Therefore,

$$
G(E, t, \theta)=\frac{\cos \theta}{L(E)} \int_{E}^{E+\Delta(E, \delta, \theta)} F\left(E_{i}, t, \theta\right) d E_{i}
$$

To obtain the TED of proton flux arriving at the TF, the effect of TOF has to be again taken into account. The distance between $d A_{1}$ and $d A_{2}$ is $p$; its explicit expression can be obtained from Eq. (4) as

$$
\begin{aligned}
\mathrm{p}^{2}= & \left(D \sin \alpha+\mathrm{R}_{2} \cos \theta_{2} \cos \alpha-\mathrm{R}_{1} \cos \theta_{1}\right)^{2} \\
& +\left(\mathrm{R}_{2} \sin \theta_{2}-\mathrm{R}_{1} \sin \theta_{1}\right)^{2} \\
& +\left(D \cos \alpha-\mathrm{R}_{2} \cos \theta_{2} \sin \alpha\right)^{2} .
\end{aligned}
$$

The solid angle subtended by $d A_{2}$ with respect to $d A_{1}$ is $d A_{2} \cos B / p^{2}$, where $\beta$ is the angle between $\vec{p}$ and $\vec{D}$. Letting $P(E, t)$ be the TED of total proton current arriving at the $\mathrm{TF}$, then

$$
P(E, t)=2 \int_{0}^{r_{1}} R_{1} d R_{1} \int_{0}^{\pi} d \theta_{1} \int_{0}^{r_{2}} R_{2} d R_{2} \int_{0}^{2 \pi} d \theta_{2} G\left(E, t-\frac{p}{v(E)}, \theta\right) \cos \beta / p^{2}
$$

The purpose of using the TF is to truncate the proton flux below a certain energy. Therefore, those protons with energy not far above the threshold suffer significant 
loss of energy. In this case, the assumption that each proton travels an approximate straight pathlength is no longer valid. There is no simple way to calculate the proton energy loss in the TF without resorting to computer processing. In the following sections, we are interested in the total energy spectrum and the TF is not used. For the discussion of the geometry given in Sec. 2 , it is to be understood that instead of having a TF, one has instead a bare detector.

\section{APPROXIMATIONS}

In the case where the source neutron flux is a very narrow pulse in time, we can approximate it by a $\delta$-function, i.e.,

$$
S_{0}(E, t)=f(E) \delta(t)
$$

From Eq. (8) and Eq. (13) we then have

$$
F(E, t, \theta)=N \frac{d \sigma}{d \Omega}\left(E_{0}, \theta\right) f\left(E_{0}\right) \delta\left(g\left(E_{0}, t\right)\right) / \cos ^{2} \theta,
$$

where $\mathrm{E}_{0}=\mathrm{E} / \cos ^{2} \theta$ and

$$
g\left(E_{0}, t\right)=t-\left[d / v\left(E_{0}\right)\right] ;
$$

$\delta(g)$ can be expressed as

$$
\begin{aligned}
\delta\left(g\left(E_{0}, t, j\right)\right. & =\delta\left(E_{0}-E_{t}\right) / \frac{\partial g}{\partial E_{0}} \\
& =\frac{m}{d} v^{3}\left(E_{t}\right) \delta\left(E_{0}-E_{t}\right)
\end{aligned}
$$

with $E_{t}$ being determined from the solution of $g\left(E_{t}, t\right)=0$, namely,

$$
E_{t}=\frac{m}{2}\left(\frac{d}{t}\right)^{2}
$$

Substituting Eq. (30) into Eq. (23) and using Eq. (27), we get 


$$
G(E, t, \theta)=\frac{m N}{d L} \frac{\cos \theta}{(E)} \frac{d \sigma}{d \Omega}\left(E_{t}, \theta\right) f\left(E_{t}\right) v^{3}\left(E_{t}\right),
$$

for $E_{t} \cos ^{2} \theta-\Delta(E, \delta, \theta) \leq E \leq E_{t} \cos ^{2} \theta ;$ and

$$
G(E, t, \theta)=0 \quad \text { otherwise. }
$$

Notice that the time-dependence of $G$ is implicitly contained in $E_{t}$, whereas the energy dependence is solely carried by $L(E)$.

If the area of the CF and the detector are much smaller than $D^{2}$ so that the angular variation in $\theta$ of $\vec{p}$ can be neglected, with $p=D, \beta=0$, and $\ddot{\theta}=\alpha$, the integrals in Eq. (25) can be trivially done

$$
P(E, t)=\left(\frac{\pi r_{1} r_{2}}{D}\right)^{2} G\left(E, t-\frac{D}{v(E)}, \alpha\right) .
$$

The detector current is then

$$
\begin{aligned}
I(t) & =e \int_{0}^{\infty} P(E, t) d E \\
& =e \int_{E_{1}(t)}^{E_{2}(t)} P(E, t) d E .
\end{aligned}
$$

The limits $E_{1}(t)$ and $E_{2}(t)$ stem from the fact that for a given $t, G(E, t, \alpha)$ is nonzero only in a limited energy range given by Eq. (3la). It follows that $E_{1}(t)$ and $E_{2}(t)$ satisfy

$$
E_{1}=\frac{1}{2} m\left(\frac{d \cos \alpha}{t-D / v\left(E_{1}\right)}\right)^{2}-\Delta\left(E_{1}, \delta, \alpha\right)
$$

and

$$
E_{2}=\frac{1}{2} m\left(\frac{d \cos \alpha}{t-D / v\left(E_{2}\right)}\right)^{2}
$$


The last equation can be readily solved as

$$
E_{2}(t)=\frac{1}{2} m\left(\frac{d \cos \alpha+D}{t}\right)^{2}
$$

However, Eq. (34) rannot be solved exactly and we resort to the following approximation scheme: We first rewrite $\mathrm{Eq}$. (34) as

$$
\frac{\mathrm{d} \cos }{v\left(\mathrm{E}_{1}\right) t-\frac{\alpha}{\mathrm{D}}}=\left(1+\frac{\Delta\left(\mathrm{E}_{1}, \ddot{0}, \alpha\right)}{\mathrm{E}_{1}}\right)^{1 / 2}
$$

Notice that $\delta$ is usually very thin so that in the range of energies of interest, $\Delta(E, \delta, \alpha) / E \ll 1$ is valid. Keeping terms only up to the first order in $\Delta / E$ in Eq. (37), we get

$$
v\left(E_{1}\right) \simeq \frac{d \cos \alpha+D}{t}\left[1-\frac{\Delta\left(E_{1}, \delta, \alpha\right)}{2 E_{1}}\left(\frac{1}{1+D / d \cos \alpha}\right)\right]
$$

Since $D / d \cos \alpha \ll 1$, a solution $v\left(E_{1}\right)$ good up tc the first order in both $\Delta / E_{1}$ and $D / d \cos \alpha$ is

$$
v\left(E_{1}\right) \simeq \frac{d \cos \alpha+D}{t}\left(1-\frac{\Delta\left(E_{1}, \delta, \alpha\right)}{2 E_{1}}\right) \text {. }
$$

Within the same order of accuracy, Eq. (39) gives

$$
E_{1}(t)=E_{2}(t)-\Delta\left(E_{1}, \delta, \alpha\right)
$$

The explicit expression for the integral in Eq. (33) is

$$
I(t)=n \cos \alpha \int_{E_{1}(t)}^{E_{2}(t)} \frac{d E}{L(E)} \frac{d \sigma}{d \Omega}\left(E^{*}(t, E), \alpha\right) f\left(E^{*}(t, E)\right) v^{3}\left(E^{*}(t, E)\right),
$$


where $n \equiv \frac{e N m}{d}\left(\frac{\pi r_{1} r_{2}}{D}\right)^{2}$ and

$$
E^{*}(t, E)=\frac{1}{2} m\left(\frac{d}{t-D / v(E)}\right)^{2}
$$

Let $E=E_{2}(t)+\varepsilon(t)$, since $E_{1} \leq E \leq E_{2}$ and $E_{2} \gg \Delta\left(E_{2}, \delta\right) \geq \varepsilon$, we can expand $E^{*}(t, E)$ around $E_{2}$

$$
\begin{aligned}
E^{*}(t, E) & =E^{*}\left(t, E_{2}\right)+\varepsilon \frac{\partial E^{*}}{\partial E_{2}}\left(t, E_{2}\right) \\
& =\frac{E_{2}}{\cos ^{2} \alpha}-\frac{1}{\cos ^{3} \alpha} \frac{D}{d} \varepsilon .
\end{aligned}
$$

The second term in Eq. (43) is second-order smaller than the first sincs $D / d \ll 1$, so we are quite justified to drop it in the integrand of Eq. (41).

$$
\begin{aligned}
I(t) & =\eta \cos \alpha \frac{d \sigma}{d \Omega}(E(t), \alpha) f(E(t)) v^{3}(E(t)) \int_{E_{1}(t)}^{E_{2}(t)} \frac{d E}{L(E)} \\
& =\eta \delta \frac{d \sigma}{d \Omega}(E(t), \alpha) f(E(t)) v^{3}(E(t)),
\end{aligned}
$$

where $E(t)=E_{2}(t) / \cos ^{2} \alpha$.

That the integral above is equal to $\delta / \cos \alpha$ follows from Eq. (22) and Eq. (40). To obtain the explicit form for the function $f(E)$, we set $E=E_{2}(t) / \cos ^{2} \alpha$ and use Eq. (36) to solve $t$ in terms of $E$ :

$$
t=\frac{d \cos \alpha+D}{v(E) \cos \alpha}
$$

Using Eq. (45) in Eq. (44), we finally obtain 


$$
f(E)=I\left(\frac{d \cos \alpha+D)}{v(E) \cos \alpha}\right) / n \delta \frac{d \sigma}{d \Omega}(E, \alpha) v^{3}(E) .
$$

This formula allows us to compute the energy spectrum of the source neutrons from the detecior current. We want to emphasize that the result in Eq. (46) is accurate to the first order in $\Delta / E$ as well as in $D / d$.

VII. SOURCE FUNCTION WITH SEPARABLE TIME- AND ENERGY-DEPENDENCE

Since the measured detector current is only a function of time, it is obvious that we cannot unfold the data to recover the TED of a neutron source in genera1. It is possitis, however, to retrieve information about the source function if its time- and energy-dependence are separable and if the time-dependence can be measured. If the time variation of the sransmission is small, the energy spectrum of the source neutron flux can, indeed, be considered as independent of time. Thus we can write

$$
S_{0}(E, t)=f(E) g(t)
$$

where $g(t)$ is nonzero only for $t_{1} \leq t \leq t_{2}$. We can rewrite $S(E, t)$ as

$$
S_{0}(E, t)=\int_{t_{1}}^{t_{2}} f(E) g\left(t^{\prime}\right) \delta\left(t-t^{\prime}\right) d t^{\prime} .
$$

From the result of the last section, the detector current resulting from a neutron source specified by $f(E) \delta\left(t-t^{\prime}\right)$ is given by Eq. (44).

$$
I\left(t-t^{\prime}\right)=n \delta \frac{d \sigma}{d \Omega}\left(E\left(t-t^{\prime}\right), \alpha\right) f\left(E\left(t-t^{\prime}\right)\right) v^{3}\left(E\left(t-t^{\prime}\right)\right)
$$

The detector current resulting from a neutron source described by Eq. (48) is therefore 


$$
I_{g}(t)=\int_{t_{1}}^{t_{2}} g\left(t^{\prime}\right) I\left(t-t^{\prime}\right) d t^{\prime}
$$

The function $g(t)$ usually can be determined by measuring the prompt $\gamma$-rays produced along with the fission neutrons. The reason for this can be understood as follows: Since the number of $\gamma$-photons are proportional to the number of fission neutrons produced at any given time, it follows that the $\gamma$-ray flux is described by the same $g(t)$ as the neutron flux, except with a different energy spectrum function. In reality, of course, we can only expect this to hold approximately since the transmission of the neutrons is not identical to that of the $\gamma$ rays. With this in mind, let us write

$$
S_{\gamma}(E, t)=q(E) g(t)
$$

to denote the time-energy dependence of the $\gamma$-ray flux. Denoting the energydependent response function of the $\gamma$-ray detector by $R(E)$, the detector current is given by

$$
I_{\gamma}(t)=\int_{0}^{\infty} E q(E) g(t) R(E) d E=g(t) \int_{0}^{\infty} E q(E) R(E) d E
$$

So up to an overall normalization, $g(t)$ is determined by $I_{\gamma}(t)$.

We note that Eq. (50) is a convolution of $g$ and $I$, hence, the function $I$ can be unfolded by the standard method of Fourier transforms. For any function $p$, let $p^{F}$ stands for its Fourier transform, i.e.,

$$
p^{F}(k)=\frac{1}{\sqrt{2 \pi}} \int_{-\infty}^{\infty} e^{i k t} p(t) d t
$$

Taking the Fourier transform of $\mathrm{Eq}$. (50), we have

$$
I^{F}(k)=I_{g}^{F}(k) / g^{F}(k) \sqrt{2 \pi}
$$


Taking the inverse Fourier transform of Eq. (54) gives

$$
I(t)=\frac{1}{2 \pi} \int_{-\infty}^{\infty} \frac{I^{F}(k)}{g^{F}(k)} e^{-i k t} d k
$$

Once $I(t)$ is obtained, we can use Eq. (46) to compute $f(E)$, and this completely determines $s_{0}(E, t)$.

\section{ACKNOWLEDGMENTS}

We would like to thank Leon Forman for useful discussions on various experimental limitations. 